\title{
Corta reseña de la historia de la artroplastia de cadera
}

\author{
Short review of the history of hip arthroplasty \\ José de Jesús González Mercado, ${ }^{*}$ Bernardo Ramírez Serratos* \\ *Médico residente de Ortopedia y Traumatología del Centro Médico Nacional de Occidente, México.
}

\begin{abstract}
Resumen
La historia en la medicina es la base para construir el futuro. En esta corta reseña se narra la genealogía de la artroplastia, planteando sus inicios como cirugías agresivas secundarias a infecciones articulares, pasando por osteotomías, evolucionando a los primeros intentos de usar material no humano como sustituto de componentes humanos, los primeros diseños anatómicos y estudios biomecánicos, hasta llegar a la sustitución total de la articulación con elementos biocompatibles de mayor durabilidad.
\end{abstract}

Palabras clave: Artroplastia total de cadera, aspectos históricos, historia.

Abstract

Medicine history is the basis for building the future. This review contains arthroplasty history, its beginnings as an aggressive surgery secondary to articular infections, passing through osteotomies, evolving to the first attempts to use non-human material as a substitute for human components, the first anatomical designs and biomechanical studies, until reaching total articular replacement with biocompatible components of grater durability.

Keywords: Total hip arthroplasty, historical aspects, history.

La historia es un profeta con la mirada vuelta hacia atrás: por lo que fue, y contra lo que fue, anuncia lo que será.

Eduardo Galeano. Escritor y poeta uruguayo.

\section{Introducción}

Los antecedentes hablan de la mitad inicial del siglo XIX, previo al descubrimiento de la anestesia, siendo los padecimientos de cadera tratados con analgésicos y antiinflamatorios. A partir de la inducción de la anestesia en 1847, la cirugía comienza sus primeros pasos, no obstante, incluso los cirujanos más agresivos solían evitar los procedimientos por las altas tasas de infección. Posterior al año 1865, cuando Lister introduce el cambio de antisepsia por asepsia, y las infecciones quirúrgicas se reducen de manera muy significativa, pero lenta, los cirujanos co-

Correspondencia:

Dr. José de Jesús González Mercado

Av. Revolución Núm. 1858, Col. Universitaria, 44840 Guadalajara, Jalisco, México.

E-mail: dr.jesusgonzalez26@gmail.com mienzan a tomar la decisión de realizar intervenciones quirúrgicas electivas.

La apertura de la posibilidad de la cirugía renueva el tratamiento de las enfermedades de cadera tomando como principal estandarte el combate a la tuberculosis de dicha región anatómica. Posterior a la década inicial del siglo XX y la Primera Guerra Mundial, la esperanza de vida comienza a crecer, lo que trae consigo nuevas enfermedades articulares y por ende nuevos tratamientos quirúrgicos para éstas. ${ }^{1}$

Múltiples autores dividen la historia de la artroplastia, a fin de facilitar su estudio, en cinco fases históricas: "osteotomía, artroplastia de interposición, artroplastia reconstructiva, artroplastia de sustitución y artroplastia total de cadera».?

La memoria de la cirugía de cadera inicia en 1729 con Sauveur François Morand (Figura 1) un estudiante y cirujano de la Charité de París, quien

Recibido: 22-02-2021. Aceptado: 11-04-2021.

Citar como: González MJJ, Ramírez SB. Corta reseña de la historia de la artroplastia de cadera. Orthotips. 2021; 17 (2): 112-120. https:// dx.doi.org/10.35366/99906 
plantea esta amputación, que en realidad sería una desarticulación, por extirpar la articulación completa, sin embargo, no era una intervención quirúrgica común porque, a pesar de su gran descripción literaria, los conceptos éticos y los riesgos elevados eran una gran contraindicación. En 1824, Astley Cooper hace referencia a un caso de amputación de cadera exitoso en un hombre de 40 años quien sufría de una infección crónica en tal articulación. En la década de 1860, durante la Guerra Civil estadounidense, las desarticulaciones de cadera fueron en aumento en campo de batalla, aunque la mortalidad demostraba ser muy alta. ${ }^{1}$

El inicio de las osteotomías se da con una cirugía que no era de cadera; esta etapa puede ser relacionada a una publicación de Charles White en 1769, quien describe el caso de un adolescente de 14 años de edad, el cual presentaba una infección en su hombro izquierdo por probable tuberculosis. Este joven fue tratado con un drenaje del absceso, aseo quirúrgico y resección del tejido necrótico. Sorpresivamente, para los contemporáneos de White el resultado fue

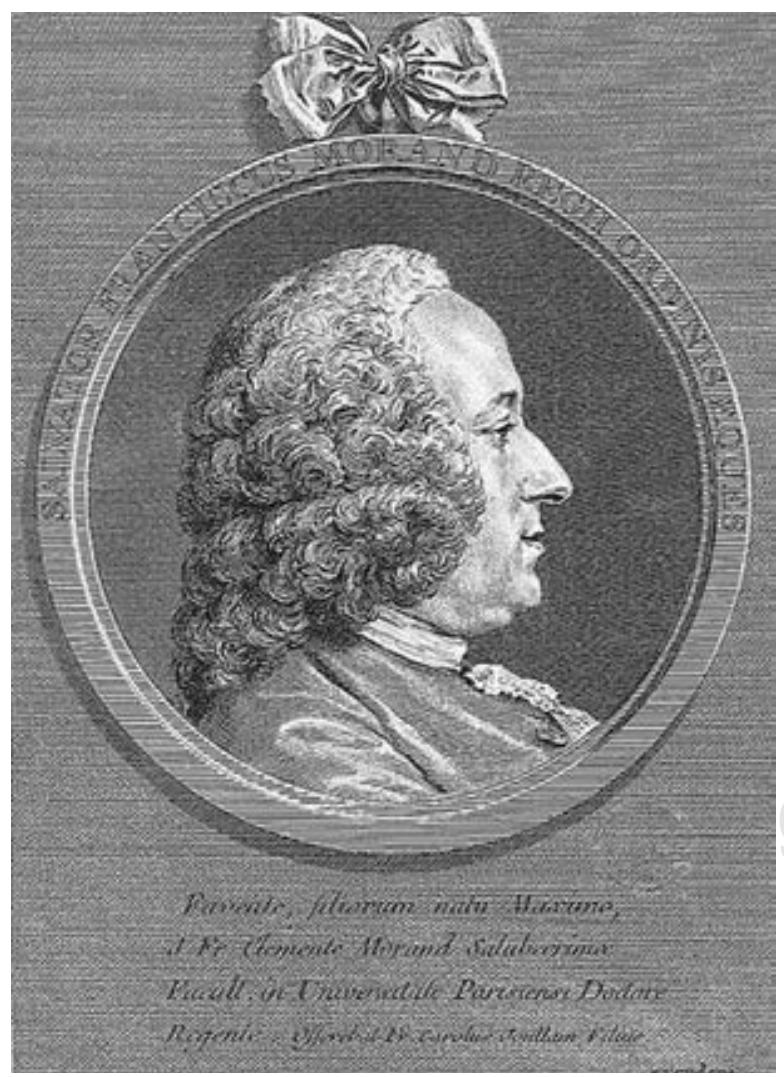

Figura 1: Sauveur François Morand (1667-1773).

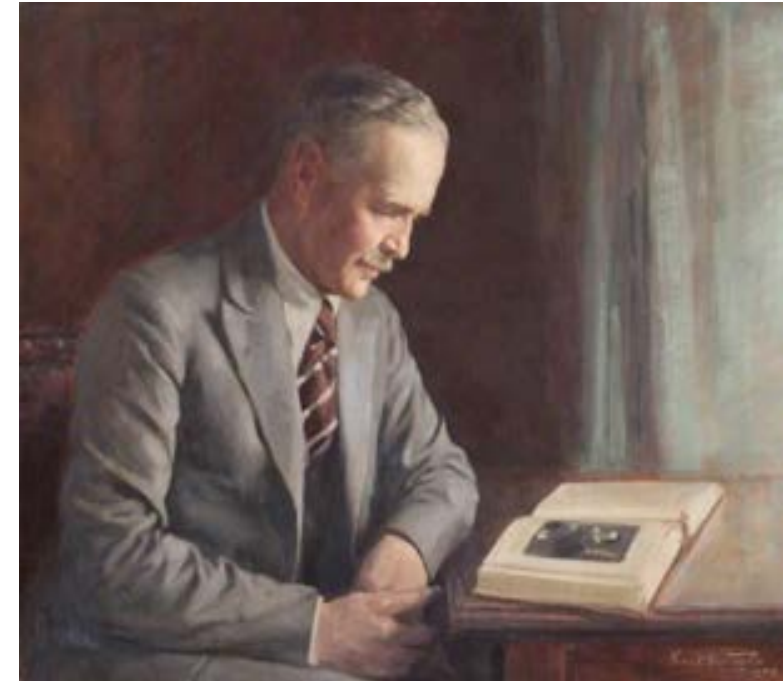

Figura 2: Robert Girdlestone (1881-1950).

favorable y el paciente recuperó gran parte de su movilidad. Gracias a este descubrimiento de White, los cirujanos comenzaron a utilizar las osteotomías debridantes como una opción de tratamiento a las infecciones articulares $y$, en 1822, Anthony White realiza una osteotomía de la articulación de cadera por un absceso por infección articular con recuperación ligera de la movilidad influenciada por otros padecimientos agregados. ${ }^{1}$

Esto da la pauta para el inicio de la primera etapa, la cual tiene un gran avance en 1832 cuando el cirujano Bernhard Heine crea la "osteotomía en cadena». Esta cirugía fue ampliamente aceptada por su facilidad de realización y buenos alcances, amplia cantidad de cirujanos hicieron estudios sobre los efectos de tal intervención. En 1874, Von Langenbeck describe el abordaje posterior de cadera que en años siguientes sería popularizado por Moore. ${ }^{1}$ El más relevante de los cirujanos mencionados es Robert Girdlestone (Figura 2), quien en 1940 crea una osteotomía la cual sigue siendo utilizada en muchas ocasiones e incluso aún se discute su uso como última opción a osteotomías infectadas. ${ }^{1}$ Inclusive dentro del siglo $\mathrm{XXI}$ siguen existiendo análisis sobre el uso de esta operación o sus variantes posteriores.

Las etapas previamente mencionadas no son secuenciales con fecha exacta sino que, como la mayoría de los adelantos en la ciencia, algunos descubrimientos son superpuestos cronológicamente debido al tiempo y dedicación de estudios de cada uno. 
En 1880 se inicia la segunda etapa con Verneuil, quien intenta las primeras artroplastias por interposición; utilizando partes blandas adyacentes para esto, y tres años después $M$. Ollier seguiría dicha rama dando una nueva pauta a la cirugía de cadera. ${ }^{3,4} \mathrm{JB}$ Murphy desarrollaría técnicas mediante el uso de fascia y grasa como tejido de interposición, $, 5,6$ algunos años después se experimentaría con láminas cromadas de vejiga de cerdo por William S Baer, quien fuese el fundador del Department of Orthopaedics del Johns Hopkins Medical School. Esta técnica seguiría siendo experimentada con diferentes tejidos por múltiples cirujanos; Colonna utilizó la cápsula como tejido de interposición en niños y Kallio probaría el uso de tejido dérmico como membrana interpuesta. ${ }^{1}$

Durante 1912 Jones comienza a usar otros materiales en las artroplastias de interposición usando láminas de oro, no obstante, los resultados continuaban siendo malos. ${ }^{7}$

En 1923 aparece el primer antecedente de una prótesis en copa llamada «artroplastia de molde» de N. Smith-Petersen (Figura 3), el concepto de esta intervención sólo se concentraba en la cabeza femoral, exponiendo el hueso esponjoso y colocando una copa sobre ella. Los primeros implantes fueron de cristal, los cuales en pocos meses se rompían, posteriormente utilizó la celulosa, sin embargo, dio reacciones de cuerpo extraño, le siguieron el uso de pirex y baquelita. Al llegar 1938, se describe el primer material metálico biocompatible: vitalium, el cual se compone de una aleación cromo-cobalto-molibdeno. ${ }^{1,6}$ En el

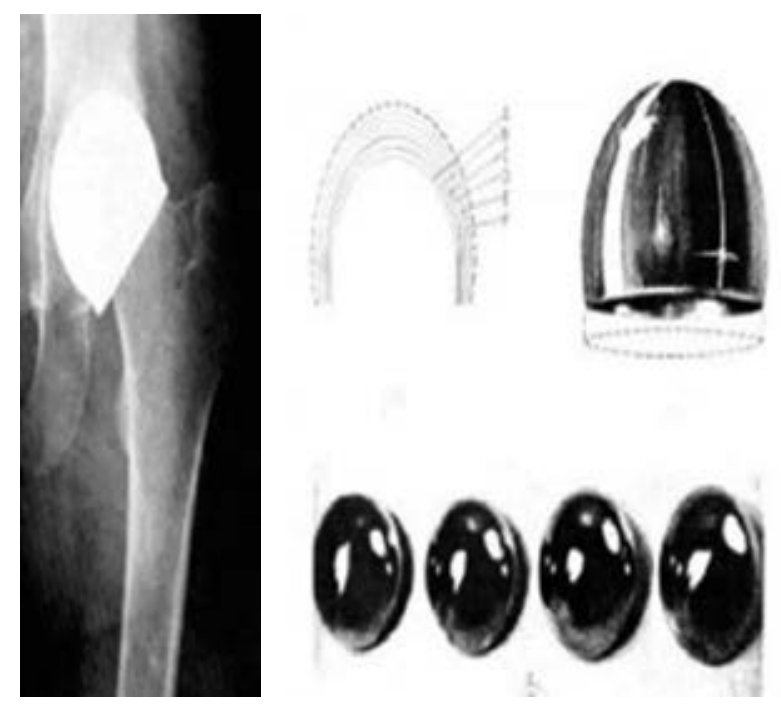

Figura 3: Copa de molde de vitalium Smith-Petersen.

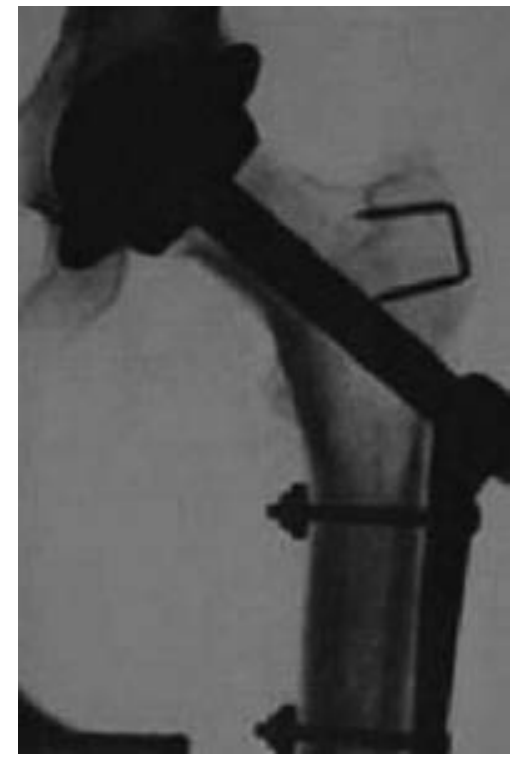

Figura 4:

Prótesis de Philip Wiles.

año 1939 Smith-Petersen presenta su técnica, ${ }^{8}$ en 1947 presenta la evolución de esa cirugía, ${ }^{9}$ y posteriormente presentaría mayor cantidad de resultado ${ }^{10}$ e incluso presentaría la mencionada técnica como alternativa para fracturas, ${ }^{11}$ pero el tiempo pasaría, hasta 1957 cuando Aufranc publicaría un artículo con 1,000 casos de caderas operadas con buenas consecuencias, con la técnica previamente mencionada. ${ }^{12}$

La tercera etapa, al igual que otras etapas, inicia gracias a las referencias de cirugías realizadas en otras regiones anatómicas: en el siglo XIX, mediante experimentación animal, Themistocles Gluck llegó a la conclusión de que el cuerpo humano podía resistir el uso de materiales no orgánicos durante largo tiempo y realizó diseños de rodillas utilizando marfil, las cuales eran fijadas con una especie de cemento compuesto por resinas con yeso o piedra pómez, ${ }^{1}$ el cual en un futuro sería la base de la creación del cemento acrílico fraguado en frío de Charnley. ${ }^{7}$ En 1903 y en 1923 se realizan artroplastias parciales de cadera sustituyendo la cabeza femoral por piezas de marfil, ${ }^{2}$ siguiendo el concepto previamente planteado por Gluck.

En 1938, en Londres se realiza la primera sustitución de cadera de componentes emparentados con acero inoxidable en seis pacientes con enfermedad de Still por parte de Philip Wiles (Figura 4). ${ }^{13}$ Él presenta una prótesis bicompartimental la cual se basaba en un elemento de cabeza femoral compuesto por un tallo, una placa lateral y tornillos y una unidad acetabular que se estabilizaba con un tornillo, siendo la prótesis no cementada primigenia; sin embargo, para Wiles la 
Segunda Guerra Mundial se interpuso en el desarrollo de su técnica y materiales. En los años 40 se crea la primera prótesis parcial utilizada por parte de Bohlman y Moore (Figura 5) en un paciente que había tenido una resección femoral proximal por tumor de células gigantes, era una pieza de endoprótesis de vitalio, con buena respuesta de $75 \%$ de movilidad. ${ }^{14}$

En esta década inicia la cuarta etapa gracias a Robert y Judet quienes diseñan una prótesis parcial de acrilico, ${ }^{1}$ la cual hacia 1952 ya tenía gran cantidad de difusión y una revisión de los primeros 600 casos. $^{12}$

En ese momento la investigación comienza ampliarse a muchos cirujanos, quienes buscan perfeccionar la artroplastia; en 1950 Moore y Reyman diseñan una endoprótesis femoral de vitalio, el cual sin necesidad de adhesivo podía anclarse al fémur ${ }^{1} y$ un par de años después presentarían un artículo con los buenos logros de ésta. ${ }^{15}$ Fred Thompson (Figura 6) diseña su hemiprótesis, la cual sigue siendo utiliza-

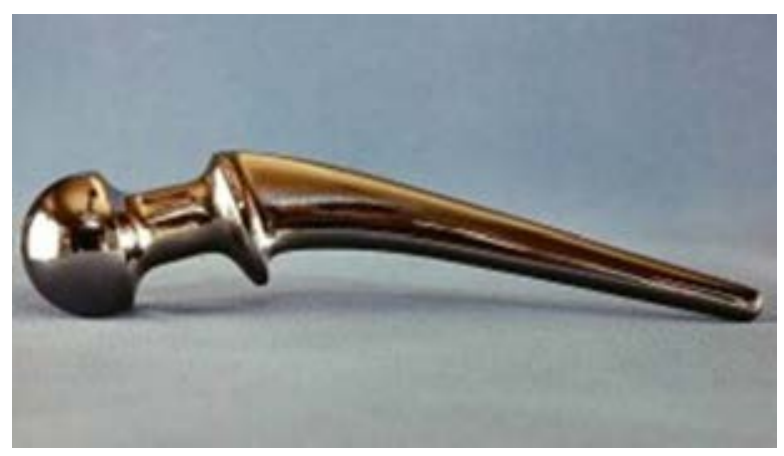

Figura 5: Hemiprótesis de Moore.



Figura 6: Hemiprótesis de Thompson.

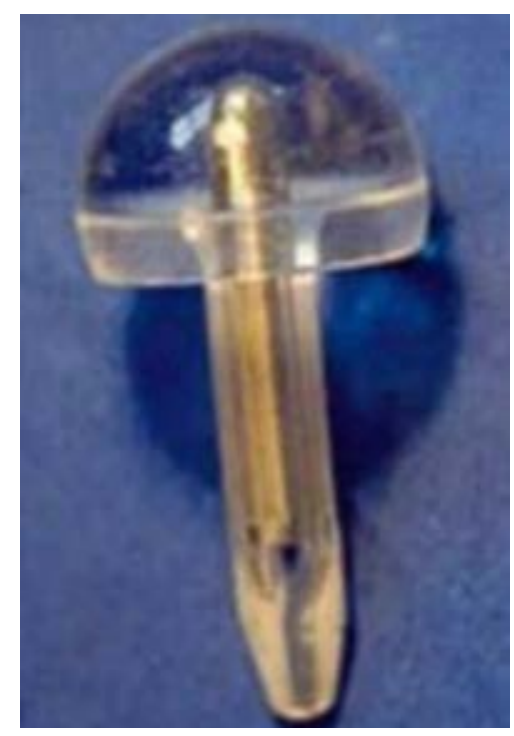

Figura 7:

Prótesis de Judet.

da hasta el presente, la cual consiste en un vástago femoral corto y curvo de vitalium, el cual necesita cemento para ser fijado. En 1952 también presentaría un artículo respaldando el uso de dicha prótesis como alternativa. ${ }^{16,17}$ Merle d'Aubigné (Figura 7) evaluaría el desarrollo de la técnica de Smith-Petersen en $1949^{18}$ siguiendo esta evaluación, y junto a Gosset y Lange Rettig en 1954 diseñarían otra prótesis con la variante de que la cabeza femoral no era metálica, utilizando metilmetacrilato. ${ }^{1}$ En los siguientes tres y cuatro años publicarían resultados sobre el uso de su prótesis en fracturas ${ }^{18}$ y movilidad posterior. ${ }^{19,20}$

A partir de este principio, GK McKee crea una nueva prótesis bicompartimental; utilizando como componente femoral un prototipo muy parecido al usado por Thompson y creando un cotillo que se ancla al trasfondo acetabular con púas creadas de acero ${ }^{21}$ que posteriormente acabaría remplazada por una aleación de cromo-cobalto. Este diseño fue modificado en conjunto con Farrar (Figura 8), evolucionando el elemento femoral por uno con el cuello más delgado. En 1966 el mismo McKee presentaría una revisión de sus casos con $90 \%$ de éxito usando la prótesis bicompartimental ${ }^{21}$ e incluso en 1973 publicaría una última serie donde sólo $1 \%$ de los casos presenta malos efectos. ${ }^{22}$ A su vez, Ring (Figura 8) en Gran Bretaña publica en 1968 el uso de una prótesis que, al sustituir ambos elementos, tiene una inherente estabilidad, esa prótesis no necesitaba cemento acrílico, se podía realizar en ambos lados al mismo tiempo e incluso se hablaba de un periodo de cuatro años con buenos desenlaces. ${ }^{23}$ 
Los modelos de cadera bicompartimentales de metal-metal continuaron apareciendo en diferentes países, sin embargo, pasaron a ser obsoletas por malos resultados, los cuales incluían incidencia alta de dolor y aflojamiento temprano, pero tal experimentación daría la pauta para el desarrollo de las prótesis totales al demostrar que la sustitución de ambos componentes tenía un mejor efecto.

La quinta etapa tiene sus inicios en la década de los cincuenta cuando John Charnley (Figura 9) prueba una nueva prótesis; el elemento femoral de acero con una cúpula de mucho menor tamaño y la pieza acetabular de teflón fijada por metilmetacrilato (quien popularizaría el uso de éste, basado en estudios de Haboush ${ }^{24}$ ), llamada «prótesis de baja fricción». Esta prótesis fracasó con el uso de teflón y fue sustituido por el polietileno de alta densidad y peso molecular. ${ }^{25,26}$ Charnley publica en 1960 un artículo como antesala a su prótesis, el cual hablaba sobre la evolución hasta esos años de la cirugía de cadera ${ }^{27}$ y en 1961 publica «Arthroplasty of the hip. A new operation» cambiando por completo la historia de las prótesis mostrando muy buenos logros a corto plazo. ${ }^{27}$ Lamentablemente, los estudios posteriores a medio y largo plazo darían malos resultados, presentando muchas reacciones adversas por el uso del cemento. ${ }^{28}$

A partir de este punto la crónica de la investigación de la artroplastia total de cadera cambiaría de forma trascendental, ya que al llegar a un punto de buenas consecuencias quirúrgicas se concentraría en el desarrollo biomecánico; la revolución de los materiales comenzaría.
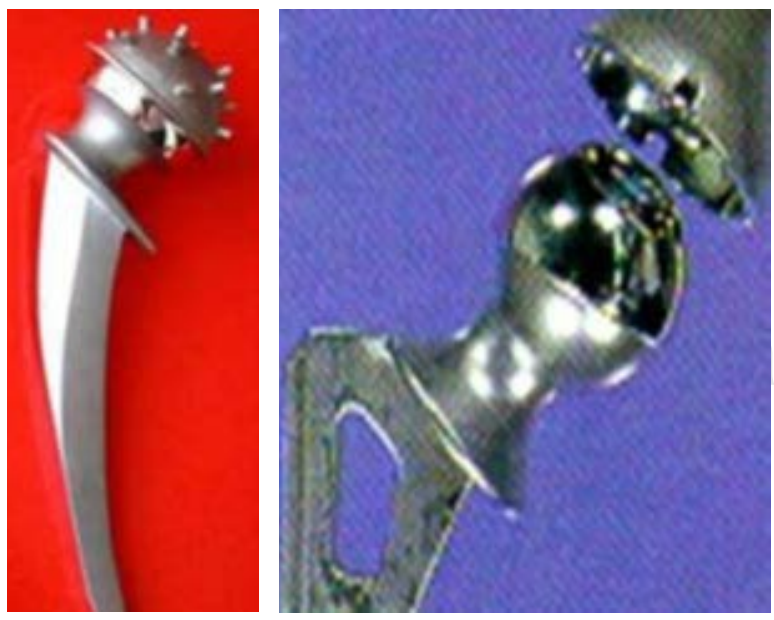

Figura 8: Prótesis de McKee-Farrar y prótesis de Ring.

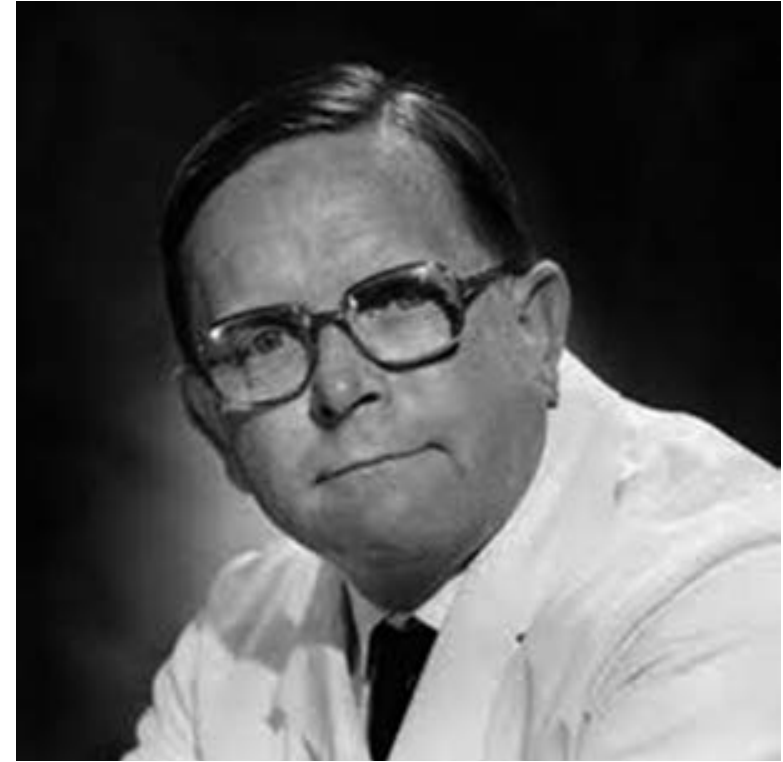

Figura 9: John Charnley (1911-1982).

Este cambio iniciaría con Frederick Pauwels quien estudia los principios biomecánicos de la cadera: fuerza de tensión, compresión, cizallamiento y rotación, también estudiaría las características biomecánicas de los principales materiales, ${ }^{29}$ resistencia, elasticidad y desgaste.

A partir de este punto se divide la narración en múltiples investigaciones de material, la evolución de la cementación se basaría en cuatro generaciones diferentes de cementación y su relación con el diseño protésico.

En 1953, Sven Kiar describiría el uso de un plástico autopolimerizante capaz de unir una prótesis al hueso, importando la técnica del área de odontología. ${ }^{30}$

La primera evaluación del cemento y su biocompatibilidad sería previa a muchos de los avances mencionados previamente. Increíblemente, el primer estudio publicado sobre esto llegaría en 1957 por Wilse, Hall y Stenehjem experimentando con conejos y monos. ${ }^{31}$

La generación original consistía en prótesis de aleaciones de cobalto, aluminio y vitalium ya mencionado, el vástago era afilado en sus bordes y el adhesivo se introducía de forma anterógrada con la mano; lo que daba una distribución no uniforme del cemento y una repartición marcada de cargas en ciertos puntos del diseño de la prótesis, lo que hizo que los resultados a corto plazo como ya fue mencionado eran excelentes, pero los de medio y 


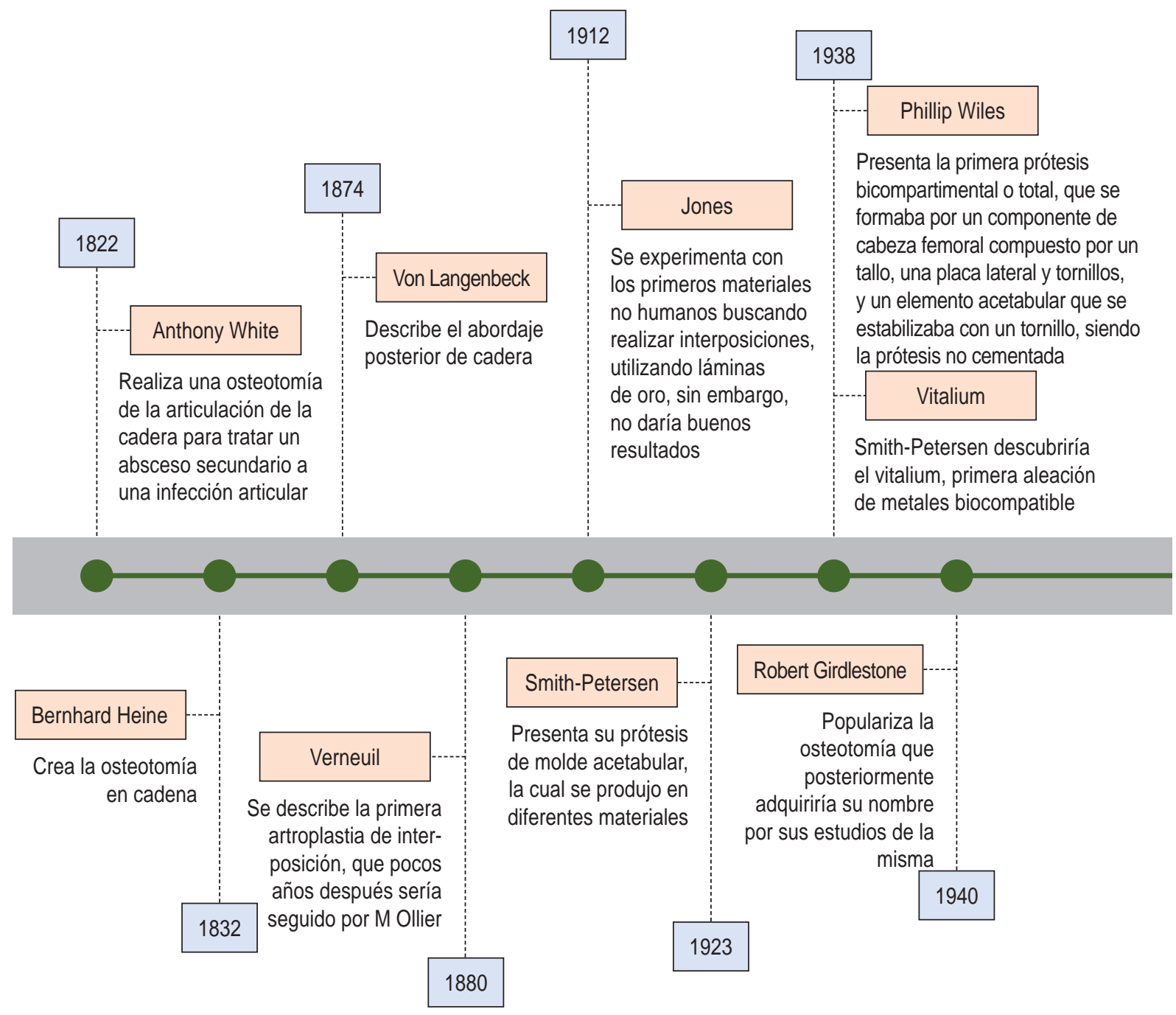

Figura 10: Línea de tiempo de artroplastia. ${ }^{1}$

largo plazo fueron devastadores, con un porcentaje alto de aflojamiento y aún más alto de revisiones por múltiples factores. ${ }^{32}$

Durante la segunda generación se intentarían combatir estos problemas: se comenzó a distribuir el cemento de forma retrograda con un previo lavado extenso de la cavidad medular, también se colocaba por vez inicial un tapón distal que mejoraría la presión del vástago y se eliminarían los bordes afilados de este mismo. Los primeros logros daban una importante mejoría, presentarían una tasa de aflojamiento menor y en mayor tiempo, no obstante, no alcanzaría a ser suficiente. ${ }^{32}$

La tercera generación se basaba en crear superficies rugosas cubiertas de polimetilmetacrilato y en reducir la porosidad del adhesivo a fin de aumentar la sujeción de ambos, los resultados fueron mejores, presentados por Pillar y Galante, y a pesar de ello se crearía una generación más buscando eliminar complicaciones. ${ }^{1,32}$

La cuarta generación sería para buscar la homogenización de las capas de cemento, se comenzaron a colocar posicionadores distales y proximales que mantenían en posición el vástago, mostrando una increíble tasa de aflojamiento de $1 \%$ en 15 años. ${ }^{32}$

En 1970, Boutin inicia con el uso de cerámica de aluminio en las artroplastias creando una prótesis de alúmina-alúmina; tomando en cuenta que este material ya era usado en odontología. ${ }^{1}$ Esta rama crearía nuevas prótesis no cementadas que disminuyen su 
fricción por las características de su material y no por la dependencia de otros materiales de unión. ${ }^{33}$ Le seguirían el uso de múltiples materiales como zirconia, hidroxiapatita, alúmina, biovidrios y otros polímeros.

El inicio de la década de los setenta traería consigo la primera prótesis bipolar gracias a Bateman y posteriormente, en 1985, Giliberty seguiría esa investigación dando como conclusión un híbrido entre la hemiprótesis y la prótesis total, el cual tenía ventajas como la disminución de la erosión articular, el aflojamiento de tallo y luxaciones. ${ }^{34}$

En 1981, Watson y Jones introducirían el abordaje anterolateral, disminuyendo el riesgo de luxación y sólo un año después Bauer presentaría el abordaje lateral directo con facilidad de técnica y una disminución importante de luxaciones. ${ }^{1}$

La investigación también se ramificaría en eliminar el adhesivo intentando evitar las complicaciones de la cementación, las prótesis se dividirían en dos grandes tipos: cementadas y no cementadas, estas últimas basadas en un vástago que ajusta a presión, con cubierta porosa de hidroxiapatita. ${ }^{7}$

Los malos resultados de las prótesis cementadas en jóvenes llevaron a continuar la rama de las artroplastias no cementadas de la mano de Judet y sus colaboradores, siendo el final de los años

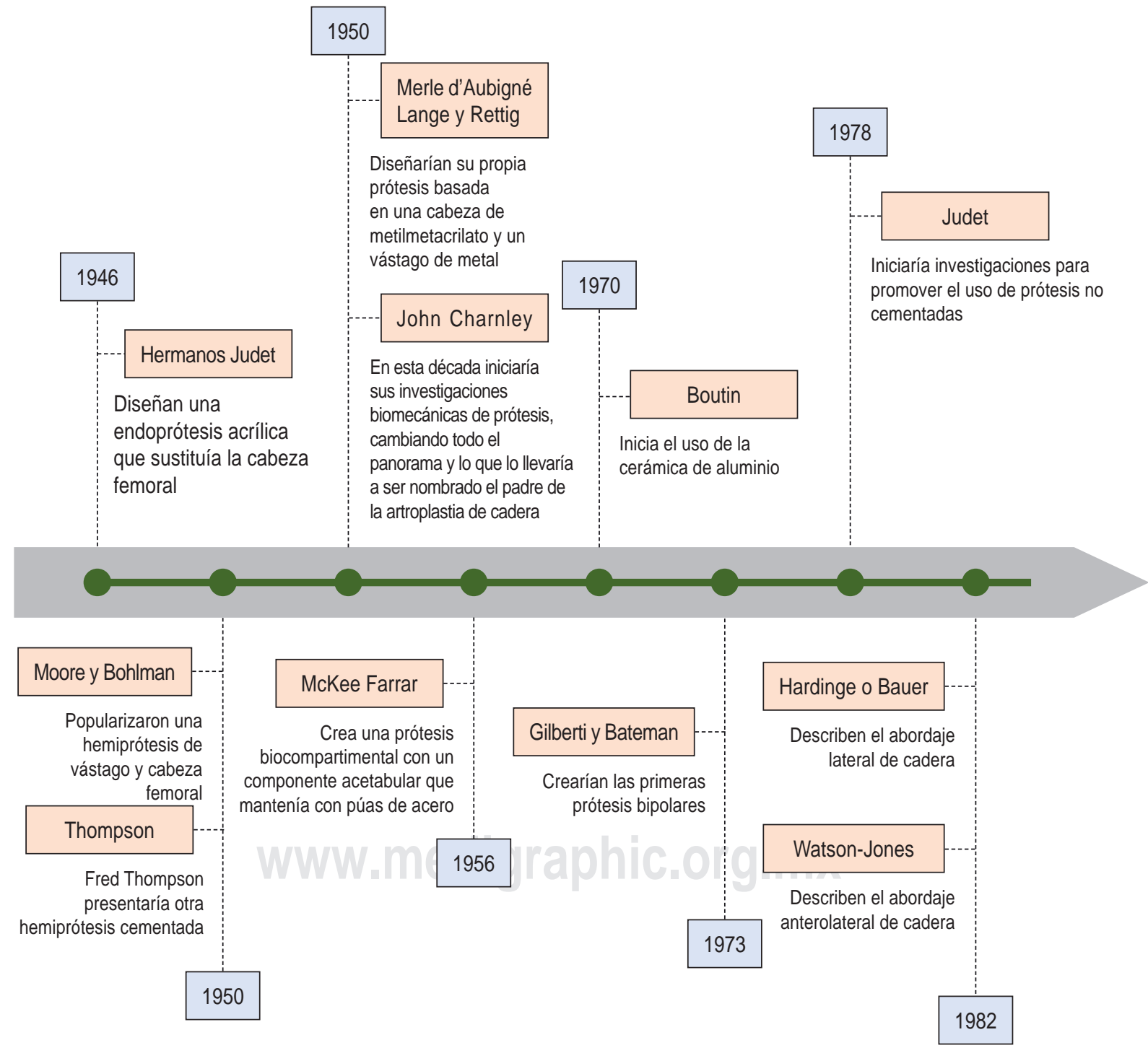

Figura 11: Línea de tiempo de artroplastia. ${ }^{2}$ 
setenta la época de nacimiento de estas mismas, tomando como prioridad la fijación y ajuste en el canal medular femoral evaluado por autores como Noble. ${ }^{35}$ En años posteriores se evaluaría la anatomía y características biomecánicas de desgaste del mismo, apareciendo en la literatura el término de "stress shielding" que se refiere a la osteopenia causada por la eliminación de la tensión típica por un implante en un hueso. ${ }^{36} \mathrm{En}$ los años subsecuentes se buscaría enfrentar este problema mediante múltiples creaciones de nuevos vástagos en la década de los dos mil: vástagos de anclaje proximal y distal que se caracterizan por el fresado de la cavidad endóstica buscando rectificar las curvaturas anatómicas del fémur, vástagos de anclaje proximal metafisario que se caracterizan por intentar un autobloqueo tipo cuña con un anclaje sólo metafisario, vástagos de anclaje anatómico que tiene como característica que es adaptado a la morfología del fémur y vástagos isoelásticos que cuentan con un módulo de elasticidad más parecido al hueso, procurando disminuir la atrofia ósea por protección de la carga. ${ }^{37}$ En los últimos años se han presentado vástagos modulares llamados «fit and fill» los cuales muestran mejores alcances en revisiones. ${ }^{38}$

Con tal de mejorar las consecuencias clínicas del reemplazo de cadera durante el próximo siglo, diversos estudios abogan por una implantación más personalizada que considere la relación cinemática de la columna lumbar/columna-cadera y la anatomía constitucional de la cadera para una artroplastia de cadera fisiológica y biomecánicamente sólida. El abordaje quirúrgico, el diseño del implante y la orientación deben depender de las características anatómicas y cinemáticas únicas del paciente. Al generar una prótesis de cadera fisiológica (a partir de la restauración anatómica del nativo) y al optimizar la interacción de los componentes durante las actividades de la vida diaria (desde la selección de una orientación de copa que se ajuste a la flexibilidad de la columna), la técnica de alineación cinemática para el reemplazo de cadera puede perfeccionar los resultados clínicos de cadera protésica. ${ }^{39}$

\section{Conclusión}

Las prótesis articulares han revolucionado la ortopedia conocida desde su creación, modificando la historia y dando una resolución a patologías que antes no tenían un gran pronóstico, a pesar de iniciar como tratamiento de otras enfermedades infecciosas, pasar por un proceso de ser la alternativa de fracturas y llegar hasta la actualidad donde son la principal alternativa de tratamiento para enfermedades crónicodegenerativas articulares.

\section{Agradecimientos}

Agradecemos a lo Doctores Aarón Piscil Magaña y Rafael Bustos Mora por su colaboración con la historia de la artroplastia y agradecemos especialmente al Doctor José María Jiménez Ávila por el incondicional apoyo para lograr la investigación necesaria y su enseñanza en este tema.

\section{Referencias}

1. Peltier LF. Historia de la cirugía de cadera. En: Callaghan JJ, Rosenberg AG, Rubash HE. Callaghan cadera osteonecrosis abordajes injerto óseo desgaste. 2a ed. Madrid: Marbán; 2012, pp. 1-27.

2. Hernandez VD. La cadera. Madrid: Editorial Médica Panamericana; 1997.

3. Ollier L. Traité des Résections et des Opérations. Conservateur quón Peut Pratiquer sur le Système Osseux (Paris). 1883.

4. Shands AR. Historical milestones in the development of modern surgery of the hip joint. En: Tronzo RG, eds. Surgery of the hip joint. Lea \& Febiger. Philadelphia, 1973.

5. Nájera CM. Reflexiones sobre la cirugía de cadera. Rev Mex Ortop Traum. 2001; 15 (1): 1-4.

6. Murphy JB. I. Arthroplasty. Ann Surg. 1913; 57 (5): 593-647. doi: 10.1097/00000658-191305000-00001.

7. Canale ST, Beaty J. Campbell cirugía ortopédica. Vías de abordaje. Marbán. 2015, 608.

8. Coventry MB. Historia de la artroplastia y los reemplazos articulares. Buenos Aires: Editorial Panamericana; Volumen 1. Reemplazos articulares. 1994. 19-21.

9. Smith-Petersen NM. Arthroplasty of the hip, a new method. J Bone Joint Surg. 1939; 21: 269-288.

10. Smith-Petersen MN. Principle of mold arthroplasty as applied to the hip. Surg Clin North Am. 1947; 27: 1303-1306.

11. Smith-Petersen MN. The classic: Evolution of mould arthroplasty of the hip joint by M. N. Smith-Petersen, J. Bone Joint Surg. 30B:L:59, 1948. Clin Orthop Relat Res. 1978; (134): 5-11.

12. Smith-Petersen MN, Larson CB. Complications of old fractures of the neck of the femur; results of treatment of vitallium-mold arthroplasty. J Bone Joint Surg Am. 1947; 29 (1): 41-48.

13. Brady OH, Masri BA, Garbuz DS, Duncan CP. Rheumatology 10 , Joint replacement of the hip and knee: when to refer and what to expect. CMA J. 2000; 163: 1285-1291.

14. Aufranc OE. Constructive hip surgery with Vitallium mold. A report on 1000 cases of arthroplasty of the hip over a 15-year period. J Bone Joint Surg. 1957; 39A: 237-248.

15. Judet $R$, Judet $J$. Technique and results with the acrylic femoral head prosthesis. J Bone Joint Surg 1952; 34-B: 173-180.

16. Moore AT. Metal hip joint: new self-locking vitallium prosthesis. South Med J. 1952; 45: 1015-1019.

17. Thompson FR. Vitallium intramedulary hip prosthesis; preliminary report. N Y State J Med. 1952; 52: 3011-3020. 
18. D’Aubigné RM, Benassy J. L'arthrodèse du poignet; Le procédé de Smith-Petersen. 1949, 1161-1163.

19. D’Aubigné RM, Postel M. Arthroplasty of the hip with acrylic prosthesis for recent and old fractures of the femoral neck. Mem Acad Chir (Paris). 1952; 78 (22-24): 695-704.

20. D'Aubigné RM, Postel M. Functional results of hip arthroplasty with acrylic prosthesis. J Bone Joint Surg Am. 1954; 36-A (3): 451-75.

21. McKee GK. Cup arthroplasty of the hip, with film. Proc R Soc Med. 1948 Jun; 41(6): 394

22. McKee GK, Watson-Farrar J. Replacement of arthritic hips by the McKee-Farrar prosthesis. J Bone Joint Surg. 1966; 48 (2): 245-256.

23. Charnley J. Anchorage of the femoral head prostheses to the saft of the femur. J Bone Joint Surg. 1960; 42B: 28-30.

24. Haboush EJ. A new operation for arthroplaty of the hip. Bull Hos Joint Dis. 1953; 14 (2): 242-247.

25. McKee GK, Chen SC. The statistics of the McKee-Farrar method of total hip replacement. Clin Orthop Relat Res. 1973; (95): 26-33.

26. Ring PA. Complete replacement arthroplasty of the hip by the ring prosthesis. J Bone Joint Surg. 1968; 50-B: 720-731.

27. Charnley J. Surgery of the hip-joint: present and future developments. Br Med J. 1960; 191 (5176): 821-826.

28. Charnley J. Arthroplasty of the hip: a new operation. Lancet. 1961; 1: 1129-1132.

29. Charnley J. The reaction of bone to self-curing acrylic cement: a long -term histologicl study in man. J Bone Joint Surg. 1970; 52-B: 340

30. Pauwels F. Biomechanichs of the normal and diseased hip. Nueva York: Springer-Verlag; 1979.

31. Wiltse LL, Hall RH, Stenehjem JC. Experimental studies regarding the posible. Use of self curing acrylic in orthopaedic surgery. J Bone Joint Surg. 1957; 49A: 961-972.
32. Peguero BA, Canales CV, Herrera RA. Artroplastia total de cadera. En: Villa Alcázar LF, Pérez Caballer AJ. Monografía médico-quirúrgica del aparato locomotor. La cadera. Tomo 1. Barcelona: Masson; 2001. 85-101.

33. Boutin P, Christel P, Dorlot JM, et al. The use of dense aluminaalumina ceramic combination in total hip replacement. J Biomed Mater Res. 1988;22 (12): 1203-1232. doi: 10.1002/jbm.820221210.

34. Noble P, Kamaric E, Alexander J, W. Distal stem centralizatio critically affects the acurate fixation of cementless femoral components. Orthop Trans. 12: 383.

35. Heredia OL, Marabini JE. Desmontaje de una prótesis bipolar. Rev Asoc Argent Ortop Traumatol. 2007; 2: 1-5.

36. Engh CA, Bobyn JD. The influence of stem size and extent of porous coating on femoral bone resorption after primary cementless hip arthroplasty. Clin Orthop Relat Res. 1988; 231: 7-28.

37. Calderer LC, Quilis AN. Cementless femoral stem in revision surgery. Preliminary results. Acta Ortopédica Mexicana. 2003; 17 (3): 122-127.

38. Suari AF, González SG, Prieto DP, García AL, Cortadellas CM, Pons MT, López FM. Impacto del hundimiento en vástagos de revisión rectos y curvos modulares en cirugía de revisión de cadera. Revista Española de Cirugía Ortopédica y Traumatología. 2017; 61 (3): 193-199.

39. Rivière C, Harman C, Logishetty K, Van Der Straeten C. Hip Replacement: Its Development and Future. In: Rivière C, Vendittoli PA, eds. Personalized Hip and Knee Joint Replacement. Cham (CH): Springer; 2020, 23-32.

\section{Conflicto de intereses}

No existe conflicto de intereses. 